\title{
NOTES
}

\section{Preparation and Monolayer Thickness of Langmuir-Blodgett Films of Polyimides Having Various Chemical Structures}

\author{
Yasunari NishiKata, Toru KoniSHI, Atsushi MoRIKAWA, \\ Masa-aki KAKIMOTO, ${ }^{*}$ and Yoshio IMAI \\ Department of Organic and Polymeric Materials, Tokyo Institute of Technology, \\ Meguro-ku, Tokyo 152, Japan
}

(Received October 7, 1987)

KEY WORDS Aromatic Polyimides / Langmuir-Blodgett Films /
Monolayer Thickness / Ellipsometry /

We have developed a method for preparing Langmuir-Blodgett (LB) films of thermally and chemically stable polyimides. ${ }^{1,2}$ The LB films were prepared by the three-step procedure shown in eq 1 , which included 1) spreading of the monolayer films of long alkylamine salts of aromatic polyamic acids 4 at the air-water interface, 2) deposition of the monolayer films of $\mathbf{4}$ on an appropriate plate, and 3) treatment of the multilayer films of 4 on the plate with a mixture of acetic anhydride and pyridine to form the multilayer films of polyimides $\mathbf{5}$.

In this paper, four kinds of LB films of 5a, $\mathbf{5 b}, 5 \mathbf{d}$, and $5 \mathbf{e}$ were newly prepared to investigate the relationship between monolayer thickness of the films and the chemical structures of the polyimides. Polyimides used in this work can be classified into three groups, a) polyimides $\mathbf{5 a}$ and $\mathbf{5 b}$ having rigid rod-like structure, b) 5c and 5d possessing flexible oxygen linkage, and c) 5e having pendant bulky phenyl groups.

\section{EXPERIMENTAL}

\section{Preparation of Polyimide LB Films}

Aromatic polyamic acids 3 were synthesized by the reaction of tetracarboxylic acid dianhydrides 1 and aromatic diamines $2^{3}$.

The solutions of $\mathbf{3}$ for monolayer spreading were prepared at a concentration of $1 \mathrm{mmol}$ $1^{-1}$ in a mixture of $N, N$-dimethylacetamide (DMAc) and benzene ( $1: 1) . N, N$-dimethylhexadecylamine 6 was dissolved in the same solvent system at the same concentration. The solutions of polyamic acid alkylamine salts 4 were prepared by mixing the solutions of $\mathbf{3}$ with that of $\mathbf{6}$ just prior to use.

The measurement of surface pressure-area isotherms ( $\pi$-A curves) of the monolayer films of 4 was carried out on a trough of $500 \mathrm{~mm}$ length and $150 \mathrm{~mm}$ width with compression speed $3.2 \mathrm{~mm} \mathrm{~s}^{-1}$ at $20^{\circ} \mathrm{C}$ using a San-Esu FSD-20 apparatus. The subphase used in the present work is deionized water.

Deposition of the monolayer films of 4 onto an appropriate plate was carried out through the air-water interface at a rate of 3$5 \mathrm{~mm} \mathrm{~min}^{-1}$ at $20^{\circ} \mathrm{C}$ by drawing upward and downward at a surface pressure of 25 and $0 \mathrm{mN} \mathrm{m}^{-1}$, respectively.

The deposited multilayer films of 4 were immersed overnight in a mixture of acetic anhydride, pyridine, and benzene $(1: 1: 3)$ to obtain the multilayer films of polyimides 5 . 


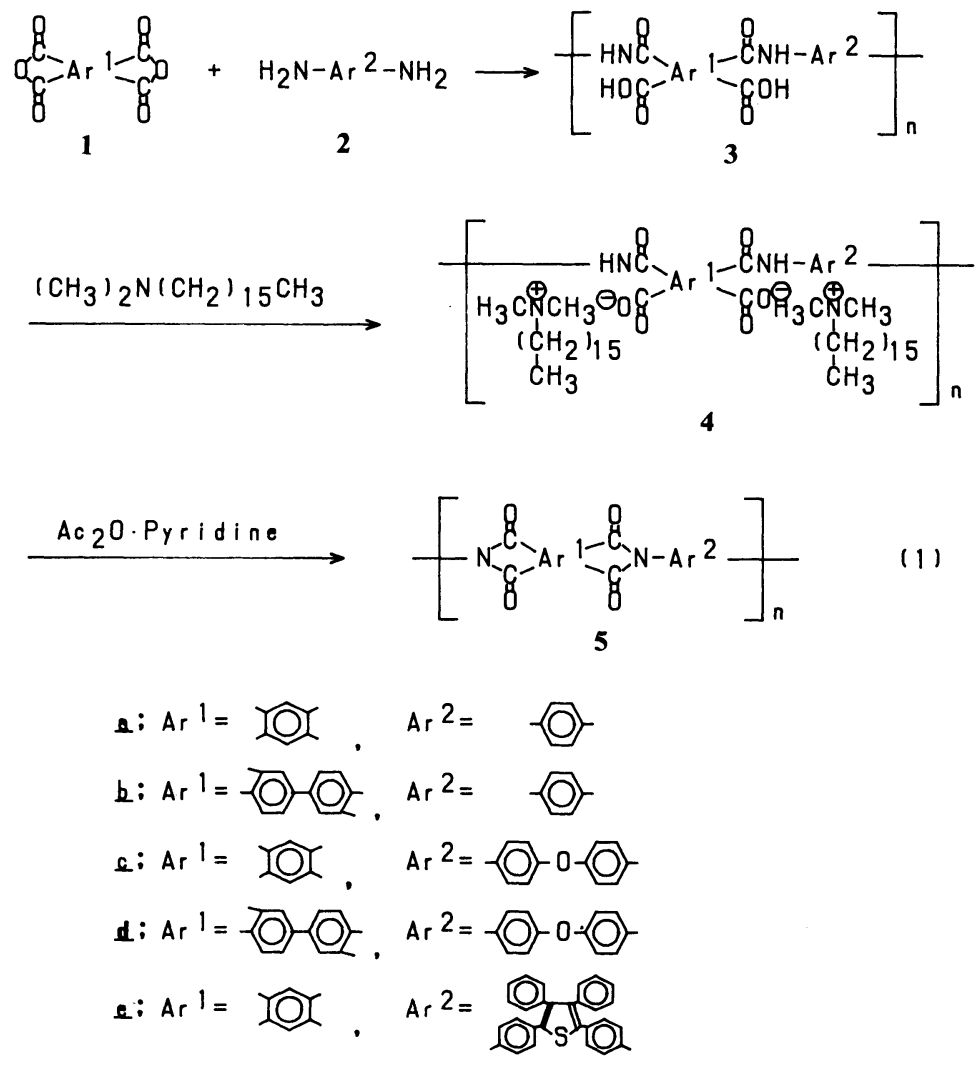

The resulting polyimide $\mathrm{LB}$ films were dried at $100^{\circ} \mathrm{C}$ under reduced pressure.

\section{Measurement}

Transmission IR spectra were measured on calcium fluoride plates where 150 layers of the film of 4 and 5 were deposited, using a LEOL FT/IR-3 spectrometer. UV spectra were recorded by a Hitachi U-3400 spectrometer, using the sample films prepared on a quartz plate. The apparatus for ellipsometry measurement was a NIIC Model EL-101, where the sample films were deposited on a silicon wafer, and they each had at least three different number of layers (typically 30, 50, and 70).

\section{RESULTS AND DISCUSSION}

The structures of the polyamic acids 3 employed are shown in eq 1 . Their molecular weights were sufficiently high so that they, except 3a, afforded flexible and tough polyimide films by usual casting method followed by heat treatment at $300^{\circ} \mathrm{C}$. However, the cast film of polyamic acid 3a having an inherent viscosity of $0.67 \mathrm{dl} \mathrm{g}^{-1}$ was very brittle, and the corresponding polyimide film 5a could not be obtained by heating the film of $3 \mathrm{a}$ at $300^{\circ} \mathrm{C}$. This characteristic is attributable to the high crystallinity of $\mathbf{3 a}$ and $\mathbf{5 a}$ as evident from the $\mathrm{X}$-ray diffraction measurement.

The behavior of the monolayer films of 4 at the air-water interface was discussed in the previous paper. ${ }^{1}$ It was suggested from the surface area-surface pressure isotherms $(\pi-\mathrm{A}$ curves) that the monolayer films were very stable. The extrapolated surface areas of 4 are listed in Table I.

Deposition of the monolayer films at the airwater interface was carried out by drawing 
Table I. Extrapolated surface area of polyamic acid alkyl amine salts 4

\begin{tabular}{cc}
\hline $\mathbf{4}$ & Surface area $/ \mathrm{nm}^{2}$ \\
\hline $\mathbf{4 a}$ & 1.1 \\
$\mathbf{4 b}$ & 1.3 \\
$\mathbf{4 c}$ & 1.4 \\
$\mathbf{4 d}$ & 1.5 \\
$\mathbf{4 e}$ & 1.4 \\
\hline
\end{tabular}

only in the upward direction to obtain Z-type multilayer films. The reason is that the transfer ratio of the downward trip was not so stable in some cases, although that of the upward trip at a surface pressure of $25 \mathrm{mN} \mathrm{m}^{-1}$ was almost 1 . The multilayer films of 4 thus obtained were converted into the multilayer films of polyimides 5 by treatment with a mixture of acetic anhydride and pyridine.

The multilayer films of both 4 and 5 were characterized by UV spectra and IR spectra. For instance, Figure 1 shows the plots of absorbance at 204 and $226 \mathrm{~nm}$ of UV spectra against the number of layers of polyamic acid alkylamine salt $4 \mathbf{a}$ and polyimide $5 \mathbf{a}$, respectively. A linear relationship obtained suggested that both films had multilayer structures. The IR spectra of $\mathbf{4 a}$ and $\mathbf{5 a}$ are depicted in Figure 2. The structure of polyamic acid long alkylamine salt $4 \mathbf{a}$ was characterized by absorptions at $2920 \mathrm{~cm}^{-1}$ (long alkyl chain), and 1660 and $1590 \mathrm{~cm}^{-1}$ (carbonyls). On the other hand, these absorptions disappeared entirely, and new characteristic absorptions due to imide carbonyl were observed at 1780 and $1720 \mathrm{~cm}^{-1}$ in the spectrum of the multilayer film of 5a. These results suggest that the cyclodehydration of $\mathbf{4 a}$ to $\mathbf{5 a}$ proceeds completely with the removal of $N, N$-dimethylhexadecylamine.

The thickness of the polyimide multilayer film 5 prepared on silican wafer was measured by ellipsometry. The results are shown in Table II. The monolayer thickness of the LB films of $5 \mathrm{a}-5 \mathrm{e}$ was found to be $0.40-0.56 \mathrm{~nm}$, and that of $5 \mathrm{c}$ agreed well with the

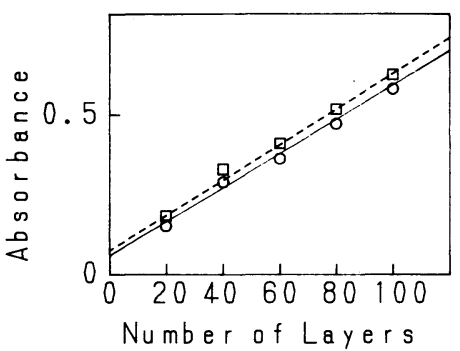

Figure 1. Plots of UV absorbance against number of layers of polyamic acid salt $4 \mathrm{a}$ at $204 \mathrm{~nm}$, and polyimide $5 \mathbf{a}$ at $226 \mathrm{~nm}$. - - - $4 \mathbf{a} ;---\square---, 5 a$.

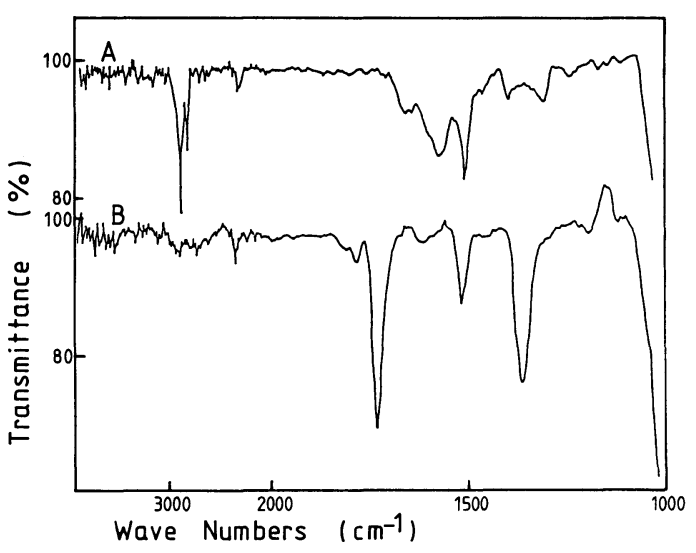

Figure 2. IR spectra of LB films of polyamic acid salt 4a (A) and polyimide 5a (B), deposited 150 layers on the $\mathrm{CaF}_{2}$ plate.

Table II. Monolayer thickness of LB films of polyimide 5

\begin{tabular}{cc}
\hline LB film & Thickness $^{\mathrm{a}} / \mathrm{nm}$ \\
\hline 5a & 0.40 \\
5b & 0.42 \\
5c & 0.45 \\
5d & 0.50 \\
5e & 0.56
\end{tabular}

a Monolayer thickness was measured by ellipsometry.

aromatic-aromatic ring facing distance calculated by X-ray measurement of the usual polyimide cast film. ${ }^{4} \mathrm{~A}$ relationship was found between the monolayer thickness and the chemical structures of polyimides 5 . The first group polyimides possessing rigid rod-like 
structures (5a and $\mathbf{5 b}$ ) have smaller values for the monolayer thickness, and the flexible ether linkage of the second group polyimides $(5 \mathbf{c}$ and 5d) made the thickness values larger. Furthermore, the pendant bulky phenyl groups apparently influenced the monolayer thickness of polyimide $\mathbf{5 e}$, making the thickness largest.

In conclusion, the LB films were successfully prepared from the simplest polyimide $\mathbf{5 a}$ whose film could not be obtained by the usual casting method via polyamic acid 3a, and from polyimide 5e possessing pendant bulky phenyl groups. The monolayer thickness of the LB films was highly dependent on chemical structure. Based on these results, the effect of introduction of some new functional groups to the polyimide LB films is under investigation.

\section{REFERENCES}

1. M. Suzuki, M. Kakimoto, T. Konishi, Y. Imai, M. Iwamoto, and T. Hino, Chem. Lett., 395 (1986).

2. M. Kakimoto, M. Suzuki, T. Konishi, Y. Imai, M. Iwamoto, and T. Hino, Chem. Lett., 823 (1986).

3. C. E. Sroog, "Macromolecular Synthesis," Coll. Vol. 1, J. A. Moore, Ed., Wiley, New York, N. Y., 1977, p 295.

4. G. Conte, L. Iliaro, N. V. Patel, and E. Giglio, J. Polym. Sci., Polym. Phys. Ed., 14, 1553 (1976). 